\title{
Role of Global and Local Strangeness Neutrality in an Inhomogeneous Freeze-Out Approach to Relativistic Heavy Ion Collisions
}

\author{
D. Zschiesche and L. Portugal \\ Instituto de Física, Universidade Federal do Rio de Janeiro \\ C.P. 68528, Rio de Janeiro, RJ 21941-972, Brazil
}

Received on 29 September, 2006

\begin{abstract}
An inhomogeneous decoupling surface of hadrons produced in relativistic heavy-ion collisions may occur, if the expanding hot and dense matter passes through a first order phase transition. We show that due to the non-linear dependence of the particle densities on the temperature and baryon-chemical potential such inhomogeneities should be visible even in the integrated, inclusive abundances. We analyze experimental data from $\mathrm{Pb}+\mathrm{Pb}$ collisions at CERN-SPS and $\mathrm{Au}+\mathrm{Au}$ collisions at BNL-RHIC to determine the amplitude of inhomogeneities and the role of local and global strangeness neutrality.
\end{abstract}

Keywords: Inhomogeneous freeze-out; Relativistic heavy-ion collisions; Global strangeness neutrality

It is expected that at sufficiently high energies, a transient state of deconfined matter with broken $Z(3)$ center symmetry and/or with (approximately) restored chiral symmetry is produced in collisions of heavy nuclei. Lattice QCD simulations [1] indicate that a second-order critical point exists, which was also predicted by effective chiral Lagrangians [2]; present estimates locate it at $T \approx 160 \mathrm{MeV}, \mu_{B} \approx 360 \mathrm{MeV}$. This point, where the $\sigma$-field is massless, is commonly assumed to be the endpoint of a line of first-order phase transitions in the $\left(\mu_{B}, T\right)$ plane. To detect that endpoint, it is hoped that by varying the beam energy, for example, one can "switch" between the regimes of first-order phase transition and cross over, respectively. If the particles decouple shortly after the expansion trajectory crosses the line of first order transitions one would expect a rather inhomogeneous (energy) density distribution on the freeze-out surface [3, 4] (similar, say, to the CMB photon decoupling surface observed by WMAP [5]). On the other hand, if the low-temperature and high-temperature regimes are smoothly connected, pressure gradients tend to wash out density inhomogeneities. Similarly, in the absence of phase-transition induced non-equilibrium effects, the predicted initial-state density inhomogeneities $[6,7]$ should be strongly damped. Thus, we investigate the properties of an inhomogeneous fireball at (chemical) decoupling. Note that if the scale of these inhomogeneities is much smaller than the decoupling volume then they can not be resolved individually, nor will they give rise to large event-by-event fluctuations. Because of the nonlinear dependence of the hadron densities on $T$ and $\mu_{B}$, they should nevertheless reflect in the average abundances.

Our basic assumption is that as the fireball expands and cools, at some stage the abundances of hadrons "freeze", keeping memory of the last instant of chemical equilibrium. This stage is refered to as chemical freeze-out. By definition, only processes that conserve particle number for each species individually, or decays of unstable particles may occur later on.

The simplest model is to treat the gas of hadrons within the grand canonical ensemble, assuming a homogeneous decoupling volume. The abundances are then determined by two parameters, the temperature $T$ and the baryonic chemical poten- tial $\mu_{B}$; the chemical potential for strangeness is fixed by the condition for overall strangeness neutrality. Fits of hadronic ratios were performed extensively $[8,9]$ within this model, sometimes also including a strangeness $\left(\gamma_{s}\right)$ or light quark $\left(\gamma_{q}\right)$ supression factor $[10,11]$ or interactions with the chiral condensate [12].

In [13] we analyzed the experimental data on relative abundances of hadrons with respect to the presence of inhomogeneities on the decoupling surface. To that end we proposed a very simple and rather schematic extension of the common grand canonical freeze-out model, i.e. a superposition of such ensembles with different temperatures and baryonchemical potentials. Each ensemble is supposed to describe the local freeze-out on the scale of the correlation length $\sim 1 / T \sim 1-2 \mathrm{fm}$. Even if freeze-out occurs near the critical point, the correlation length of the chiral condensate is bound from above by finite size and finite time effects, effectively resulting in similar numbers [14]. On the other hand, for small chemical potential, far from the region where the $\sigma$-field is critical, the relevant scale might be set by the correlation length for Polyakov loops, which is of comparable magnitude [15]. The entire decoupling surface contains many such "domains", even if a cut on mid-rapidity is performed. We therefore expect that the distributions of temperature and chemical potential are approximately Gaussian [16]. Besides simplicity, another goal of the analysis is to avoid reference to a particular dynamical model for the formation or for the distribution of density perturbations. In fact, we presently aim merely at checking whether any statistically significant signal for the presence of inhomogeneities is found, and here especially, what influence local or global strangeness neutrality, respectively, have. More sophisticated dynamical models could be employed in the future to understand the evolution of inhomogeneities from their possible formation in a phase transition until decoupling. For simplicity, and for lack of an obvious motivation for assuming otherwise, we shall take $P\left(T, \mu_{B}\right)$ to factorize into a distribution for $T$, times one for $\mu_{B}$. These distributions could, in principle, be obtained from the real-time evolution of the phase transition $[3,4]$. The densities of strange particles depend also on the strangeness-chemical potential $\mu_{S}$, which we determined in [13] by imposing lo- 
cal strangeness neutrality. However, the effect of independent fluctuations of $\mu_{S}$ should also be looked at, in particular for collisions at low and intermediate energies $\left(\sqrt{ } s_{N N} \lesssim 15 \mathrm{GeV}\right)$. This may help to reproduce the $\bar{\Lambda}$ to $\bar{p}$ ratio, which was found to be larger than one [18] and the $K^{+} / \pi^{+}$enhancement around $E_{\mathrm{Lab}} / A=30 \mathrm{GeV}$ [19]. Then the hadron abundances depend on six parameters: the arithmetic means of the temperatures and chemical potentials of all domains, $\bar{T}, \bar{\mu}_{B}$ and $\bar{\mu}_{S}$, and the widths of their Gaussian distributions, $\delta T, \delta \mu_{B}$ and $\delta \mu_{S}$. They read:

$$
\begin{aligned}
& \bar{\rho}_{i}\left(\bar{T}, \bar{\mu}_{B}, \bar{\mu}_{S}, \delta T, \delta \mu_{B}, \delta \mu_{S}\right)=\int_{0}^{\infty} d T P(T ; \bar{T}, \delta T) \\
& \int_{-\infty}^{\infty} d \mu_{B} P\left(\mu_{B} ; \bar{\mu}_{B}, \delta \mu_{B}\right) \int_{-\infty}^{\infty} d \mu_{S} P\left(\mu_{S} ; \bar{\mu}_{S}, \delta \mu_{S}\right) \rho_{i}\left(T, \mu_{B}, \mu_{S}\right),
\end{aligned}
$$

with $\rho_{i}\left(T, \mu_{B}, \mu_{S}\right)$ the actual "local" density of species $i$, and with $P(x ; \bar{x}, \delta x) \sim \exp \left[-(x-\bar{x})^{2} / 2 \delta x^{2}\right]$ the distribution of temperatures and chemical potentials within the decoupling three-volume (the proportionality constants normalize the distributions over the intervals where they are defined). In addition, strangeness conservation enters now as a global constraint for the mean of the strange chemical potential $\bar{\mu}_{S}$ :

$$
f_{s}=\sum_{i} \bar{\rho}_{i}\left(\bar{T}, \bar{\mu}_{B}, \bar{\mu}_{S}, \delta T, \delta \mu_{B}, \delta \mu_{S}\right)\left(n_{s}^{i}-n_{\bar{s}}^{i}\right)=0
$$

with $f_{s}$ the net strangeness, $n_{s}^{i}, n_{s}^{i}$ the number of strange and anti-strange quarks of hadron species $i$, respectively. In the limit $\delta T, \delta \mu_{B}, \delta \mu_{S} \rightarrow 0$ the Gaussian distributions are replaced by $\delta$-functions and the conventional homogeneous freeze-out scenario is recovered: $\bar{\rho}_{i}\left(\bar{T}, \bar{\mu}_{B}, \bar{\mu}_{S}, 0,0,0\right)=\rho_{i}\left(\bar{T}, \bar{\mu}_{B}, \bar{\mu}_{S}\right)$ and the corresponding strangeness neutrality condition fixing $\bar{\mu}_{S}$. In other words, in that limit the average densities are uniquely determined by the first moments of $T$ and $\mu_{B}$. For the present analysis we set the width of the distribution for the strange chemical potential equal to zero, $\delta \mu_{S}=0$. Since eq.(2) only ensures global strangeness neutrality, this still offers the possibility of finite net strangeness values in individual domains, in contrast to our former analysis, where we fixed $\mu_{S}$ by vanishing net-strangeness, $f_{s}=0$, locally, i.e. in each single domain. With setting $\delta \mu_{S}=0$ and the global constraint for $\overline{\mu_{S}}$, the densities again are a function of four parameters: $\bar{T}, \bar{\mu}_{B}, \delta T$ and $\delta \mu_{B}$. Our main goal here is to investigate how the fits on the experimentally measured particle abundances depend on the local or global strangeness neutraliy [20]. We compute the densities $\rho_{i}\left(T, \mu_{B}, \mu_{S}\right)$ in the ideal gas approximation, supplemented by an "excluded volume" correction:

$$
\rho_{i}\left(T, \mu_{B}, \mu_{S}\right)=\frac{\rho_{i}^{\mathrm{id}-\mathrm{gas}}\left(T, \mu_{B}\right), \mu_{S}}{1+v_{i} \sum_{j} \rho_{j}^{\mathrm{id}-\mathrm{gas}}} .
$$

This schematic correction models repulsive interactions among the hadrons at high densities. $v_{i}$ denotes the volume occupied by a hadron of species $i$; we employ $v=\frac{4}{3} \pi R_{0}{ }^{3}$ with $R_{0}=0.3 \mathrm{fm}$ for all species [17]. For all fits over the full solid angle, we fixed the isospin chemical potential by equating the total charge in the initial and final states; for the mid-rapidity fits at high energies, we fixed $\mu_{I}=0$.

To determine the four parameters of the model we minimize

$$
\chi^{2}=\sum_{i}\left(r_{i}^{\exp }-r_{i}^{\text {model }}\right)^{2} / \sigma_{i}^{2}
$$

in the space of $\bar{T}, \bar{\mu}_{B}, \delta T$, and $\delta \mu_{B}$. That is, we obtain leastsquare estimates for the parameters, assuming that they are independent. In (4), $r_{i}^{\text {exp }}$ and $r_{i}^{\text {model }}$ denote the experimentally measured and the calculated particle ratios, respectively, and $\sigma_{i}^{2}$ is set by the uncertainty of the measurement. Wherever available, we sum systematic and statistical errors in quadrature.

The data used in our analysis are the particle multiplicities measured by the NA49 collaboration for central $\mathrm{Pb}+\mathrm{Pb}$ collisions at beam energy $E_{\mathrm{Lab}} / A=20,30,40,80$ and $158 \mathrm{GeV}$ [19], and those measured by STAR for central $\mathrm{Au}+\mathrm{Au}$ collisions at BNL-RHIC, ref. [21, 22] (for individual references see [13] ref [27] and [28]). At RHIC energies, we analyze the midrapidity data; at SPS energies, we restrict ourselves to the $4 \pi$ solid angle data by NA49 in order to avoid biases arising from differing acceptance windows of various experiments. Furthermore, our checks showed that the fit results can depend somewhat on the actual selection of experimental ratios. Hence, where possible, we have opted for the least bias by choosing $r_{i}^{\exp }=N_{i}^{\exp } / N_{\pi}^{\exp }$, i.e. the multiplicity of species $i$ relative to that of pions. The specific data sets we used at the different energies are given in [13].

Where appropriate, feeding from strong and electromagnetic decays has been included in $r_{i}^{\text {model }}$ by replacing $\rho_{i} \rightarrow$ $\rho_{i}+B_{i j} \rho_{j}$. The implicit sum over $j \neq i$ runs over all unstable hadron species, with $B_{i j}$ the branching ratio for the decay $j \rightarrow i$, which were taken from [23]. From all the resonances listed by the Particle Data Group [23], mesons up to a mass of $1.5 \mathrm{GeV}$ and baryons up to a mass of $2 \mathrm{GeV}$ were included, respectively. The finite widths of the resonances were not taken into account, and unknown branching ratios were excluded from the feeding. These details are irrelevant for the qualitative behavior of $\delta T$ and $\delta \mu_{B}$ but do, of course, matter for quantitative results.

Fig. 1 shows the minimal $\chi^{2}$ per degree of freedom (taken as the number of data points minus the number of parameters) for the homogeneous approach and the inhomogeneous approach with local or global strangeness neutrality, respectively. Note that the $\chi^{2}$-values for the homogeneous model are in general agreement with the analysis done in [8] and other data from the literature $[9,10,22]$. As already shown in [13], for intermediate SPS energies, $E_{\mathrm{Lab}} / A \simeq 30-160 \mathrm{GeV}, \chi^{2} /$ dof is considerably smaller for the inhomogeneous freeze-out surface than for the homogeneous case, which is far outside the $95.4 \%$ confidence interval [24]. On the other hand, at $E_{\mathrm{Lab}} / A=20 \mathrm{GeV}$ and at RHIC energies, $\chi^{2} /$ dof is similar for the inhomogeneous with local strangeness neutrality and the homogenous model. However, between 20 and $80 \mathrm{GeV}$ the $\chi^{2} /$ dof values for the inhomogenous approach with local strangeness neutrality are still rather large (between 2 and 4). In contrast, the inhomogenoues model with global strangeness conservation gives $\chi^{2} /$ dof $\approx 1$ for $E_{\mathrm{Lab}} / A \simeq 20-160 \mathrm{GeV}$. 


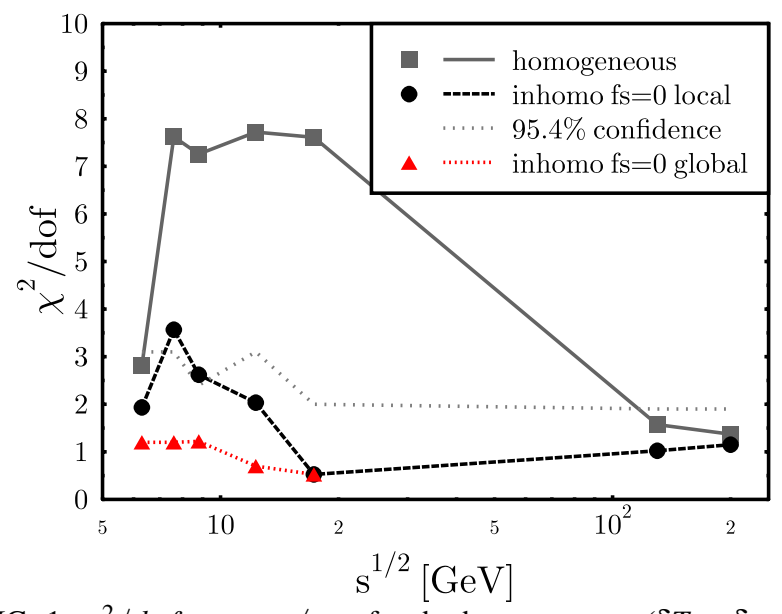

FIG. 1: $\chi^{2} /$ dof versus $\sqrt{ } s_{N N}$ for the homogeneous $(\delta T=\delta \mu=0$, squares) and the inhomogeneous fit ( $\delta T$ and $\delta \mu$ free parameters, circles and triangles). Circles denote the case of local strangeness neutrality, while triangles represent the global strangeness neutrality case. The lines are meant to guide the eye. Furthermore, the $\chi^{2} /$ dof corresponding to the $95.4 \%$ confidence interval is shown by the dotted line.

It is important to note that this result is not due to introducing an additional parameter, but just due to allowing for domains of finite strangeness with global strangeness neutrality. The calculations for RHIC energies are under way, but due to the corresponding small baryon chemical potentials at these high energies no considerable effect is expected. Thus, the inhomogenoues model gives a very satisfactory description $\left(\chi^{2} / d o f \approx 1\right)$ of the experimental data for particle abundance ratios from lowest SPS energies up to highest RHIC energies.

At RHIC the inhomogeneous model does not provide a statistically significant improvement of the description of the measured particle ratios compared to a homogeneous approach. Thus, the assumption of a nearly homogeneous decoupling surface can not be rejected there. On the other hand, the considerable improvement of the description of the data for $E_{\mathrm{Lab}} / A \simeq 30-160 \mathrm{GeV}$ indicates that at intermediate and high SPS energies the experimental data favor an inhomogeneous freeze-out surface. For the SPS $20 \mathrm{GeV}$ data the situation is not clear: there is certainly a reduction of the $\chi^{2} / d o f$ in the inhomogeneous approach, but the homogeneous model already gives a reasonable value. Here more experimental data are certainly necessary.

To illustrate the significance of inhomogeneities differently, we show contours of $\chi^{2} / d o f$ in the plane of $\delta T, \delta \mu_{B}$ in figs.2, 3 , and 4 . Here, $\bar{T}$ and $\bar{\mu}_{B}$ were allowed to vary freely such as to minimize $\chi^{2}$ at each point. Fig. 2 shows that at RHIC energy, $\chi^{2}$ is very flat in both directions. With the present data points, a homogeneous freeze-out model appears to be a reasonable approximation at high energies. In contrast, fig. 3 shows that $\chi^{2}$ is relatively flat along the $\delta \mu_{B}$ direction, while $\delta T$ is determined more accurately and is clearly non-zero. In general we find that in the approach with local strangeness neutrality there is little correlation between $\delta T$ and $\delta \mu_{B}$ and that about the minimum, $\chi^{2}$ is rather flat in $\delta \mu_{B}$ direction. Finally, fig. 3 shows the contours at SPS 158 for the case of global strangeness conservation. Now, the $\chi^{2}$ determines

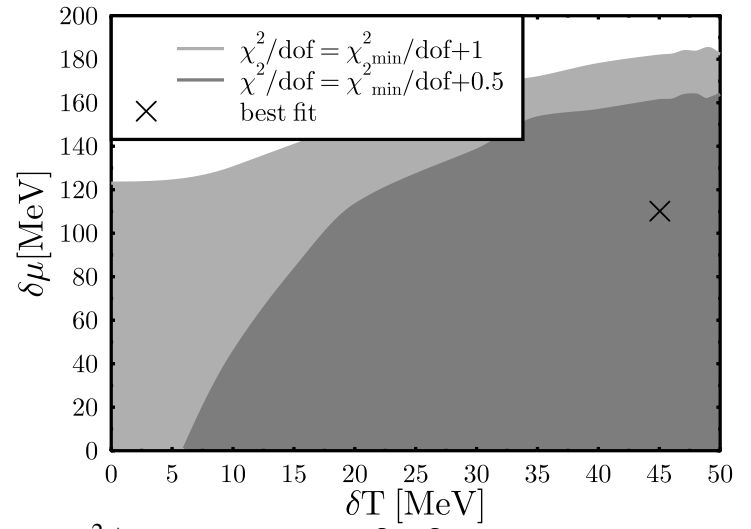

FIG. 2: $\chi^{2} / d o f$ contours in the $\delta T, \delta \mu_{B}$ plane for top RHIC energy, $\left(\sqrt{ } s_{N N}=200 \mathrm{GeV}\right)$. The other two parameters $\left(\bar{T}, \bar{\mu}_{B}\right)$ are allowed to vary freely. The $\chi^{2} /$ dof minimum is indicated by the cross.

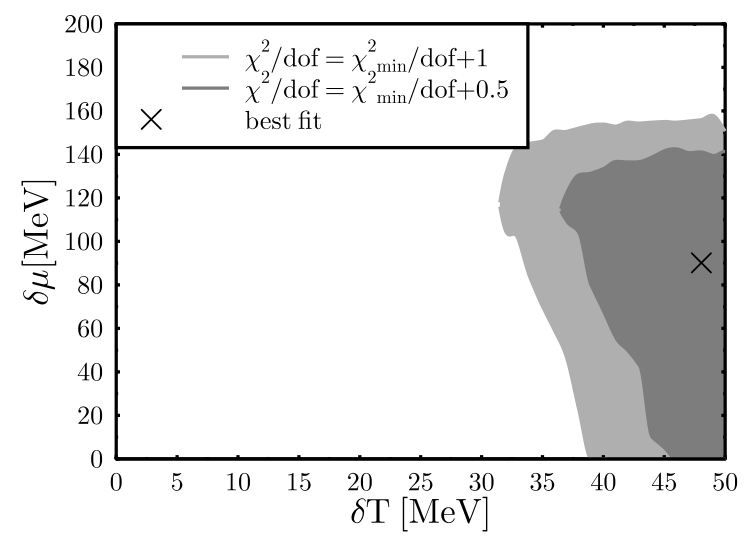

FIG. 3: Same as fig. 2 for top SPS energy $\left(E_{\mathrm{Lab}}=158 \mathrm{GeV}\right)$ with local strangeness neutrality.

the $\delta \mu_{B}$ more accurately, favoring relatively large finite values. For $\delta T$, again, values different from zero are strongly favored. The better accuracy in the width of the $\mu_{B}$-distribution

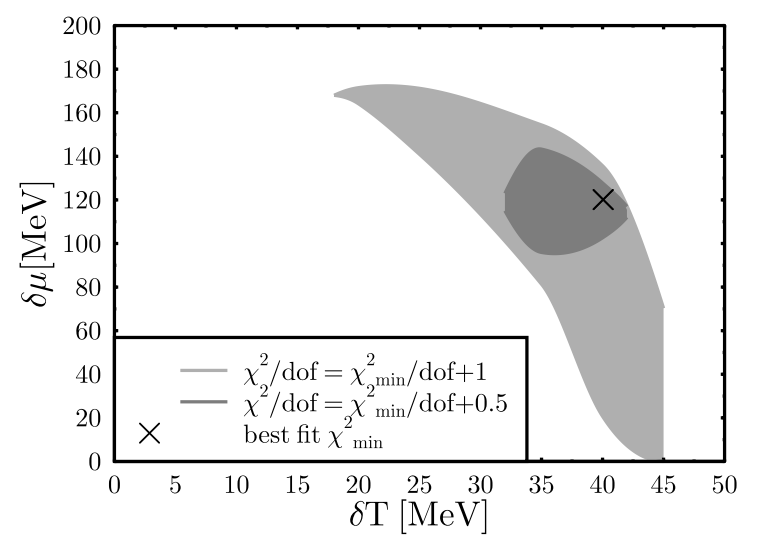

FIG. 4: Same as fig. 2 for top SPS energy $\left(E_{\mathrm{Lab}}=158 \mathrm{GeV}\right)$ with global strangeness neutrality.

is because for local strangeness conservation, the possible increased production of strange particles was restricted by the demand of vanishing strangeness and thus an accordingly adjusted strange chemical potential. This is not the case anymore if only global strangeness conservation is demanded.

In summary, we have shown that inhomogeneities on the 
freeze-out hypersurface do not average out but reflect in the $4 \pi$ (or midrapidity), single-inclusive abundances of various particle species. This is due to the non-linear dependence of the hadron densities $\rho_{i}\left(T, \mu_{B}\right)$ on the local temperature and baryon-chemical potential. Consequently, even the average $\bar{\rho}_{i}$ probe higher moments of the $T$ and $\mu_{B}$ distributions. In [13] we showed that an inhomogenoues model with local strangeness conservation strongly improves the description of the data at medium and top SPS energies compared to homogeneous approach. Here we showed that inducing global strangeness neutrality results in a further reduction of the $\chi^{2}$ at SPS energies - thus yielding $\chi^{2} /$ dof $\approx 1$ for the whole range from lowest SPS to highest RHIC energies, without adding an additional parameter. Furthermore, while for local strangeness neutrality we observed a rather flat $\chi^{2} /$ dof in $\delta \mu_{B}$ direction, this is determined more accuratrely if strangeness neutrality is ensured globally. Rather in this approach a high statistical significance for a finite width of the distributions for temperature and baryon chemical potential at medium and high SPS energies is observed.

Future studies should shed more light on whether these inhomogeneities can indeed be interpreted as fingerprints of a first-order phase transition. Eventually, one would want to establish more quantitative relations between the amplitudes of the $T, \mu_{B}$ inhomogeneities and the properties of the phase transition, e.g. its latent heat and interface tension. Furthermore, the role of inhomogeneities in the net-strangeness distribution should be studied. Data from GSI-FAIR, the low energy program at RHIC and and CERN-LHC will provide additional constraints for the evolution of chemical freeze-out with energy.

\section{Acknowledgements}

We thank C. Blume and M. Gazdzicki for helpful discussions about the NA49 data and A. Grunfeld for helping with the construction of the resonance table. The work was supported partially by CAPES and CNPq.
[1] Z. Fodor and S. D. Katz, JHEP 0404, 050 (2004).

[2] M. Stephanov, K. Rajagopal, and E. V. Shuryak, Phys. Rev. Lett. 81, 4816 (1998).

[3] D. Bower and S. Gavin, Phys. Rev. C 64, 051902 (2001).

[4] K. Paech and A. Dumitru, Phys. Lett. B 623, 200 (2005); K. Paech, H. Stöcker, and A. Dumitru, Phys. Rev. C 68, 044907 (2003).

[5] http://map.gsfc.nasa.gov/m_mm.htm]

[6] M. Gyulassy, D. H. Rischke, and B. Zhang, Nucl. Phys. A 613, 397 (1997); M. Bleicher et al., Nucl. Phys. A 638, 391 (1998); H. J. Drescher, S. Ostapchenko, T. Pierog, and K. Werner, Phys. Rev. C 65, 054902 (2002)

[7] O. J. Socolowski, F. Grassi, Y. Hama, and T. Kodama, Phys. Rev. Lett. 93, 182301 (2004).

[8] A. Andronic, P. Braun-Munzinger, and J. Stachel, Nucl. Phys. A 772, 167 (2006) [arXiv:nucl-th/0511071].

[9] see for example K. Redlich, J. Cleymans, H. Oeschler, and A. Tounsi, Acta Phys. Polon. B 33, 1609 (2002).

[10] J. Rafelski, J. Letessier, and A. Tounsi, Acta Phys. Polon. B 27, 1037 (1996); F. Becattini, M. Gazdzicki, A. Keranen, J. Manninen, and R. Stock, Phys. Rev. C 69, 024905 (2004);

[11] F. Becattini, J. Manninen, and M. Gazdzicki, arXiv:hep$\mathrm{ph} / 0511092$.

[12] D. Zschiesche, S. Schramm, J. Schaffner-Bielich, H. Stöcker, and W. Greiner, Phys. Lett. B 547, 7 (2002); W. Florkowski, W. Broniowski, and M. Michalec, Acta Phys. Polon. B 33, 761 (2002); D. Zschiesche, G. Zeeb, K. Paech, H. Stöcker, and S. Schramm, J. Phys. G 30, S381 (2004);

[13] A. Dumitru, L. Portugal, and D. Zschiesche, Phys. Rev. C 73, 024902 (2006) [arXiv:nucl-th/0511084].

[14] B. Berdnikov and K. Rajagopal, Phys. Rev. D 61, 105017 (2000); K. Paech, Eur. Phys. J. C 33, S627 (2004).

[15] A. Dumitru and R. D. Pisarski, Phys. Lett. B 504. 282 (2001); Nucl. Phys. A 698, 444 (2002).

[16] The investigation of other distributions and also the application of the "superstatistics" approach [30] are under way.

[17] P. Braun-Munzinger, I. Heppe, and J. Stachel, Phys. Lett. B 465, 15 (1999).

[18] C. Höhne (for the NA49 collaboration), nucl-ex/0510049. Our present best fit (of the $4 \pi$ ratios) for the inhomogeneous model without independent fluctuations of $\mu_{S}$ yields $\bar{\Lambda} / \bar{p}=0.93$ at $E_{\mathrm{Lab}} / A=40 \mathrm{GeV}$, for example, versus 0.76 for $\delta T=\delta \mu_{B}=0$ (without contributions from weak decays).

[19] M. Gazdzicki et al. [NA49 Collaboration], J. Phys. G 30, S701 (2004) [arXiv:nucl-ex/0403023];

[20] Calculations for finite values of $\delta \mu_{S}=0$ are under way. However, checking the influence of global strangeness conservation without adding an additional parameter represents the first step to be taken in this direction.

[21] J. Cleymans, B. Kämpfer, M. Kaneta, S. Wheaton, and N. Xu, Phys. Rev. C 71, 054901 (2005).

[22] O. Barannikova [STAR Collaboration], arXiv:nuclex/0403014; J. Adams et al. [STAR Collaboration], arXiv:nuclex/0501009; J. Adams et al. [STAR Collaboration], Phys. Rev. Lett. 92, 112301 (2004) [arXiv:nucl-ex/0310004];

[23] S. Eidelman et al. [Particle Data Group Collaboration], Phys. Lett. B 592, 1 (2004).

[24] For SPS-158 only the $4 \pi$ fit is shown; restricting the homogeneous fit to the mid-rapidity data gives smaller $\chi^{2} / \operatorname{dof}$, but still significantly higher than in the inhomogeneous approach. $\chi^{2}$ is smaller if other particle ratios are considered, as for example $\Xi / \Lambda, \Omega / \Xi$ instead of $\Xi / \pi, \Omega / \pi$ [8] or if finite widths of resonances are taken into account [11]. However, the increase of $\chi^{2}$ at SPS energies is generic if $\mathrm{Na} 494 \pi$-data are fitted.

[25] D. Zschiesche, arXiv:nucl-th/0505054, fig. 7.

[26] E. V. Shuryak and M. A. Stephanov, Phys. Rev. C 63, 064903 (2001); M. Abdel-Aziz and S. Gavin, Phys. Rev. C 70, 034905 (2004).

[27] see e.g. eq. (30) in B. L. Ioffe, I. A. Shushpanov, and K. N. Zyablyuk, Int. J. Mod. Phys. E 13, 1157 (2004);

[28] H. Heiselberg and A. D. Jackson, arXiv:nucl-th/9809013; S. J. Lindanbaum, R. S. Longacre, and M. Kramer, arXiv:nuclth/0304082; W. N. Zhang, S. X. Li, C. Y. Wong, and M. J. Efaaf, Phys. Rev. C 71, 064908 (2005).

[29] C. Markert, J. Phys. G 31, S169 (2005).

[30] C. Beck, Phys. Rev. Lett. 87, 180601 (2001); C. Beck and E. G. D. Cohen, Physica A 322, 267 (2003); 